\title{
Two-dimensional Viscoelastic Flow of Polymer Solution at Channel Junction and Branch
}

\author{
Part 2: Branch and Pocket Flow at T-Shaped Channel \\ By Taro Nishimura*, Kiyoji Nakamura**, and Akira Horikawa**,
}

\author{
Members, TMSJ
}

*Faculty of Textile Science, Kyoto Institute of Technology, Matsugasaki, Sakyoku, Kyoto 606, Japan

**Faculty of Engineering, Osaka University, Suita, Osaka 565, Japan

Based on the Journal of the Textile Machinery Society of Japan, Vol. 37, No. 2, T9-T18 (1984)

\begin{abstract}
Two-Dimensional viscoelastic flow through T-shaped channel were observed by means of flow visualization techniques. In a preceding paper ${ }^{[1]}$,the authors discussed junction flows. In the present paper additionally two types of branching flows (symmetric and asymmetric flows) and three types of pocket flows (L-shape, reverse L-shape and straight flows) through T-shaped channel were observed. Water solution of polyacrylamide was used as the viscoelastic fluid, and dextrose syrup and water were used as the Newtonian fluids.

The results are as follows. In symmetric T-shaped branch flows of viscoelastic fluids two circulating secondary flows formed on the upstream corners of the branch while with Newtonian water similar circulating flows formed on the downstream side of the two corners. A more abrupt change in the direction of flow at the branch was observed with viscoelastic fluids than with Newtonian fluids.

In the non-symmetric branch flows of viscoelastic fluids in which one branch made a 90 degrees turn while the other branch flowed straight ahead a secondary flow formed on the upstream corner at a flow rate higher than in symmetric branch flows.

Also tested were $\mathrm{L}$, reverse $\mathrm{L}$ and "straight" flows. In the $\mathrm{L}$ flow the fluid entered the lower branch of the $T$ junction with the upper right branch of the $T$ is closed to form a packet. Reverse $L$ flow was the same as L except the flow direction was reversed. The "straight" flow passed through the upper horizontal part of the $T$ while the lower branch was closed to form a pocket.

In the $\mathrm{L}$ and reverse $\mathrm{L}$ flows of viscoelastic fluids secondary flows formed on the upstream corner of the bend and in the L and "straight" flow a prominent bulge of flow into the pocket was observed. The bulge, however, diminished with increasing flow rate.

Numerical simulation of all above flows agreed reasonably with experiments except it failed to predict the bulge of flow into the pocket.
\end{abstract}

\section{Introduction}

Viscoelastic and Newtonian flows through T-shaped junction were observed experimentally and simulated numerically in a previous paper ${ }^{[1]}$ by the present authors. In $\mathrm{T}$-shaped junction flows of viscoelastic fluids, circulating secondary flows were observed experimentally at the upstream corners of the junction and flow directions were found to change more abruptly at the corner than with Newtonian fluids. Numerical simulation of viscoelastic flow using the Maxwell constitutive model in perturbation method agreed qualitatively with experimental results.

In the present study branch flows and pocket type flows in $\mathrm{T}$-shaped channels of different width were observed experimentally and simulated numerically using the Maxwell constitutive model in perturbation method.

\section{Experimental}

Shown on Fig. 1 is the T-shaped channels having dif- ferent channel width. Four combination of channel width were used: $W_{3}=10 \mathrm{~mm}, W_{1}=10 \mathrm{~mm}, 5 \mathrm{~mm}$ or $2.5 \mathrm{~mm}$ and $W_{3}=3 \mathrm{~mm}, W_{1}=10 \mathrm{~mm}$.

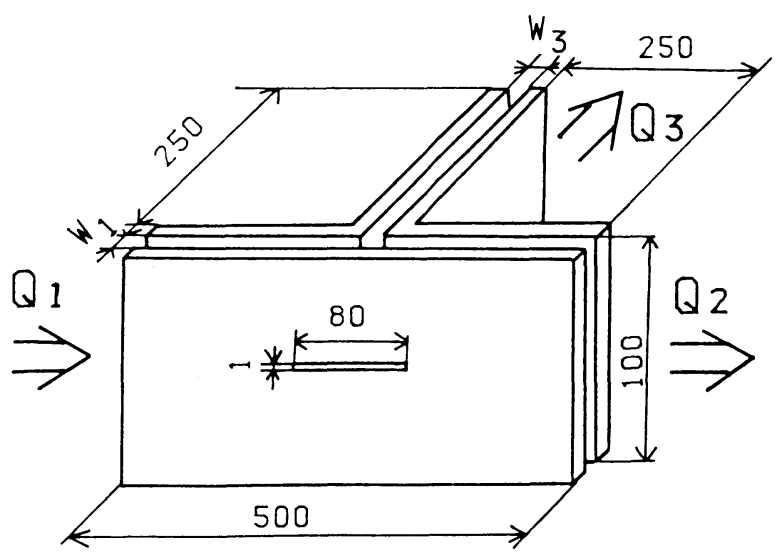

Fig. 1 Schematic of channels. 
Two kinds of branch flows and three kinds of block flows were tested using the $\mathrm{T}$-shaped channel in Fig. 1: symmetric and asymmetric branch flows and $\mathrm{L}$, reverse $\mathrm{L}$ and "straight" flows. Symmetric branch flow has negative volume flow rates $Q_{1}, Q_{3}$ respectively in channels 1 and 3 , and positive $Q_{2}$ in channel 2 with the directions of positive flow rates shown in Fig. 1. Asymmetric branch flow has positive $Q_{1}, Q_{2}$ and $Q_{3}$. Three types of pocket flows are L flow where fluid flows from channel 3 to 2 with the far end of channel 1 closed to form a packet, in reverse $\mathrm{L}$ the fluid flows from channel 1 to channel 3 with the far end of channel 2 closed and the "straight" flow is from channel 1 to channel 2 with channel 3 closed on its far end.

The viscoelastic fluid tested was a $0.8 \%$ water solution of polyacrylamide Separan AP-30 (PAA) and the Newtonian viscous fluids were dextrose syrup $1.3 \mathrm{~Pa}$. $\mathrm{s}$ in viscosity and plain water. A small amount of aluminum powder was added to the fluids as a tracer for photograph. Details of the experimental apparatus were described in a previous paper. ${ }^{[1]}$

\section{Branch Flows}

\subsection{Experimental results and discussions}

A. Streamlines in symmetric branch flows

Shown in Fig. 2 are streamlines found in symmetric branch flows. Subscripts on average flow rates $V$ and Reynolds numbers $R_{e}$ denote channel number pertaining to these values.
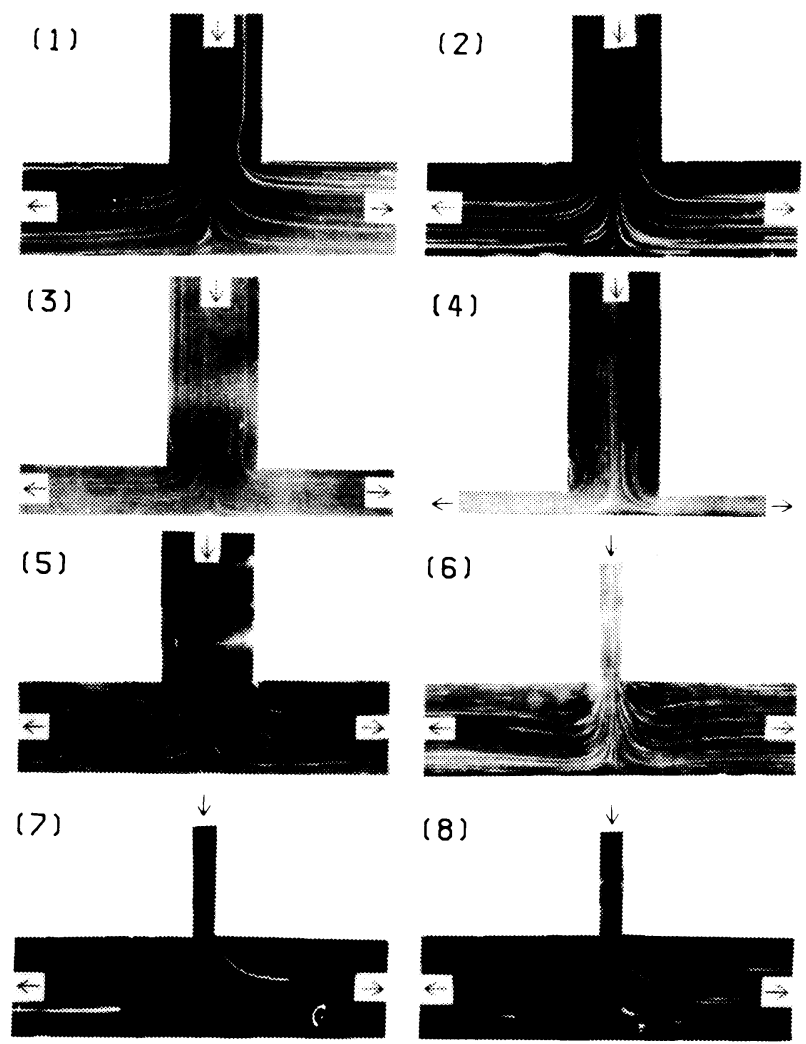

Fig. 2 Experimentally observed flow patterns for symmetric branch flows: [1] PAA, $V_{1}=V_{2}=500 \mathrm{~cm} / \mathrm{min}, R e_{1}=5.2$, [2] syrup, $V_{1}=V_{2}=25 \mathrm{~cm} / \mathrm{min}, R e_{1}=4.3 \times 10^{-2}$, [3] PAA, $V_{3}=160 \mathrm{~cm} / \mathrm{min}, R e_{3}=6.3 \times 10^{-1}$, [4] PAA, $V_{3}=120 \mathrm{~cm} /$ $\min , R e_{3}=4.4 \times 10^{-1}$, [5] water $V_{1}=V_{2}=60 \mathrm{~cm} / \mathrm{min}, R e_{1}=$ $1.0 \times 10^{2}$, [6] water $V_{1}=V_{2}=8 \mathrm{~cm} / \mathrm{min}, R e_{1}=13$, [7] PAA, $V_{1}=V_{2}=75 \mathrm{~cm} / \mathrm{min}, R e_{1}=2.3 \times 10^{-1}$, [8] syrup, $V_{1}=V_{2}=$ $2.5 \mathrm{~cm} / \mathrm{min}, R e_{1}=4.3 \times 10^{-3}$
Circulating secondary flows are observed on the upstream corners with viscoelastic fluids as shown in Fig. 2 [1]. The circulating secondary flow starts to appear at a higher flow velocity than in the merging flow observed previously. ${ }^{[1]}$ Possible reason for the delayed formation of secondary flow is that the flow is decelerated at the branch by half in branching flow while the flow is accelerated by twice in merging flow. Fig. 2[2] shows the flow lines for Newtonian fluid at a low Reynolds number. Unlike in Fig. 2[1] 'circulating secondary flow is absent.

Photographs [3] and [4] in Fig. 2 show streamlines for branching viscoelastic flow through channels of $5 \mathrm{~mm}$ and $2.5 \mathrm{~mm}$ width. In the flow of photograph [3] the branching does not change the flow speed, but in photograph [4] the downstream flow speed is twice that upstream. Photographs [1], [3] and [4] show similar circulating secondary flows at channel corners but at flow rates decreasing in the order of photographs [1], [3] and [4]. Evidently the narrower is the width to downstream channel the lower becomes the flow rate at which the secondary flow forms. Fig. 2[5] and [6] are streamlines for Newtonian water at a high Reynolds number. In this case, a secondary flow resulting from inertial effect is observed at the downstream of the branch in marked difference from the viscoelastic secondary flow forming at the upstream corner. Photographs [6], [7] and [8] in Fig. 2 show the stream lines in $3 \mathrm{~mm}$ wide upstream channel and $10 \mathrm{~mm}$ wide downstream channels. Flow is greatly decelerated in this branching flow. With Newtonian fluids streamlines change from the uniform flow in small Reynolds number to the formation of secondary flow at downstream corner of the branch. With viscoelastic fluids, streamlines tend to bend abruptly at the branch as shown in Fig. 2[8].

\section{B. Flow directions in symmetric branch flows}

Flow direction at the branch were read off the photographs [1], [2] and [3] shown in Fig. 2 at locations indicated in Fig. 3 by coordinates $\mathrm{a}, \mathrm{b}, \mathrm{c}, \mathrm{a}^{*}, \mathrm{~b}^{*}, \mathrm{c}^{*}$ etc. Also shown in Fig. 3 are coordinates $x, x^{*}$ and angles $\theta$ and $\theta^{*}$. The dotted lines are spaced a quarter of the channel width apart with lines $c, g$ and $c^{*}$ being extensions of channel walls.

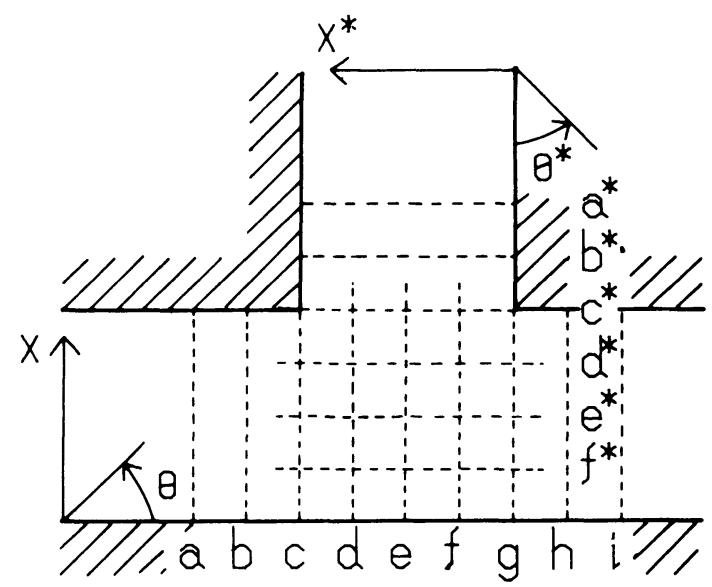

Fig. 3 Positions of flow direction measurement.

Flow directions observed in Figs. 2 as shown in Fig. 4. With Newtonian fluids flow directions on dotted lines a* and $b *$ are nearly parallel to walls while on line $c^{*}$ the flow 
changes direction toward the two exits and this trend was common to syrup with low Reynolds number and water with high Reynolds number. Put in other words $\theta^{*}$ nearly zero on line $\mathrm{a}^{*}$ and $\mathrm{b}^{*}$ while $\theta^{*}$ was positive on $\mathrm{c}^{*}$ at distances in the range of $x^{*} / W_{3}>0.5$ and negative where $x^{*} / W_{3}<0.5$. With viscoelastic fluids, however, flow direction on lines $\mathrm{a}^{*}$ and $\mathrm{b} *$ turns to the direction opposite to the branch and the change of $\boldsymbol{\theta}^{*}$ on line $\mathrm{c}^{*}$ is more abrupt than with Newtonian fluids.
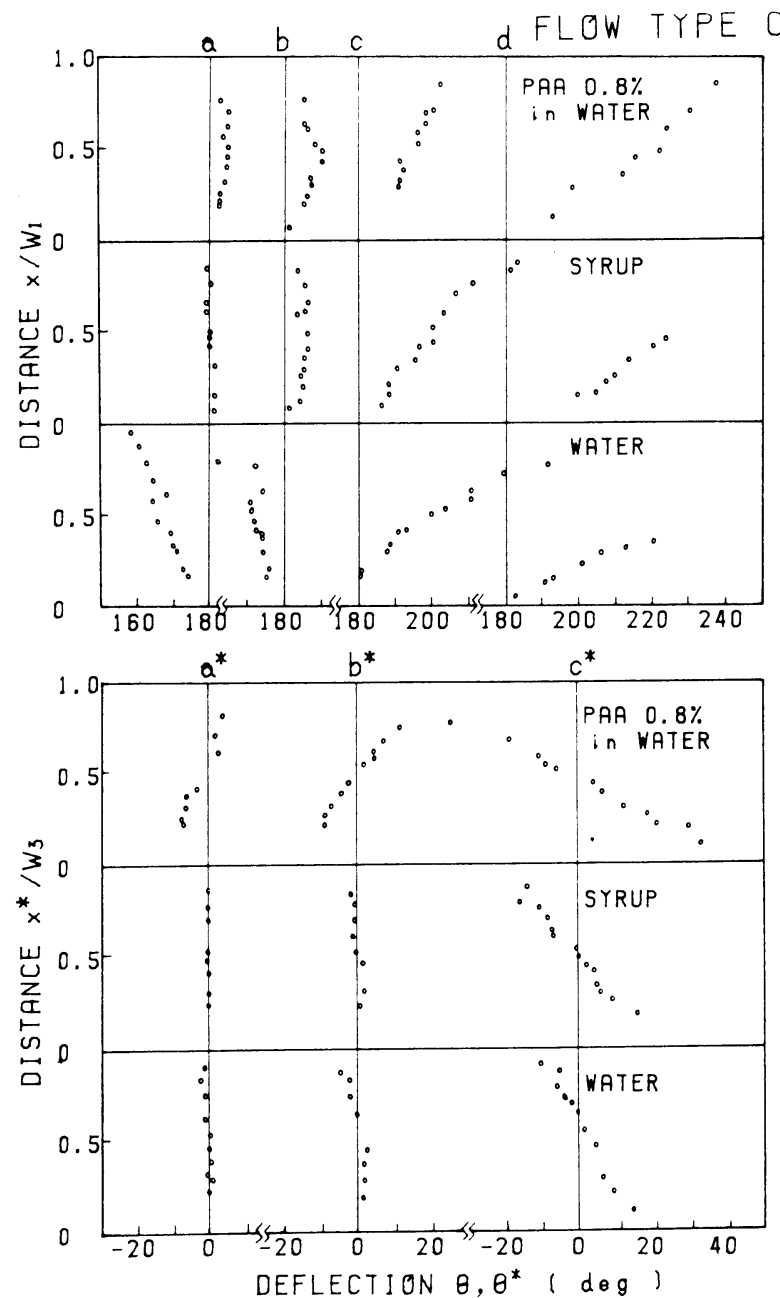

Fig. 4 Experimental flow direction distributions for symmetric branch flows.

After branching flow direction curves for PAA solution and syrup are similar to each other but the $\theta$ values for PAA solution on line $c$ are slightly smaller than those for the syrup to show that the flow of PAA solution turns more abruptly at the junction than that of syrup. The flow from narrow entrance channel 3 shown in Fing. 2[7] show similar $\theta$ values on line c. For water flow at large Reynolds number the angle $\theta$ of flow direction is smaller than $180^{\circ}$ on lines $a$ and $b$ because of the secondary flow on downstream corner.

In summary, in symmetric branch flows streamlines of viscoelastic fluids turn more abruptly than that of Newtonian fluid at the branch.

C. Streamlines in asymmetric branch flows

Experimentally observed asymmetric branch flows are shown in Fig. 5. Figure 5[1] and [2] show streamlines for viscoelastic PAA solution at large and small Reynolds number respectively.

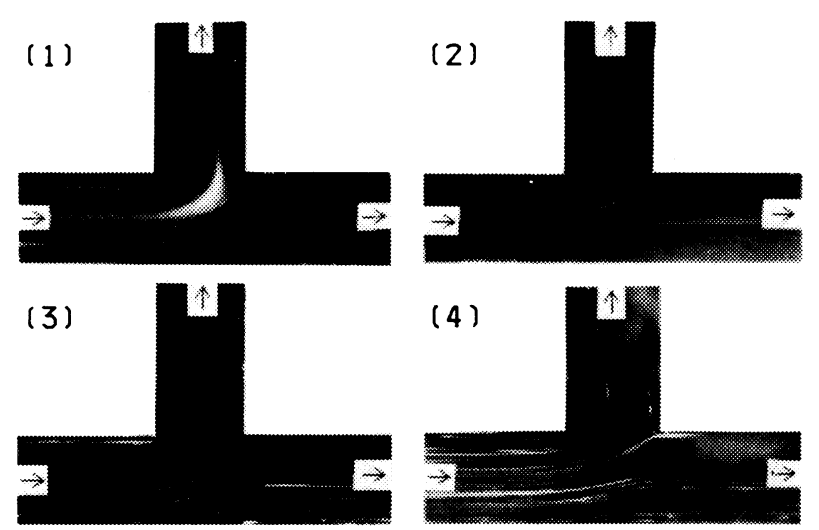

Fig. 5 Flow patterns for asymmetric branch flows: [1] PAA, $V_{2}=V_{3}=500 \mathrm{~cm} / \mathrm{min}, R e_{1}=5.2$, [2] PAA, $V_{2}=V_{3}=12$ $\mathrm{cm} / \mathrm{min}, R e_{2}=1.1 \times 10^{-2}$, [3] syrup, $V_{2}=V_{3}=2.5 \mathrm{~cm} / \mathrm{min}$, $R e_{2}=4.3 \times 10^{-3}$, [4] water, $V_{2}=V_{3}=15 \mathrm{~cm} / \mathrm{min}, R e_{2}=2.5$.

A circulating secondary flow is observed at the upstream corner. However, the secondary flow appeared at higher flow rate in asymmetric branch flow than in symmetric branch flow. This tendency was similar to the previous observations of symmetric and asymmetric merging flows

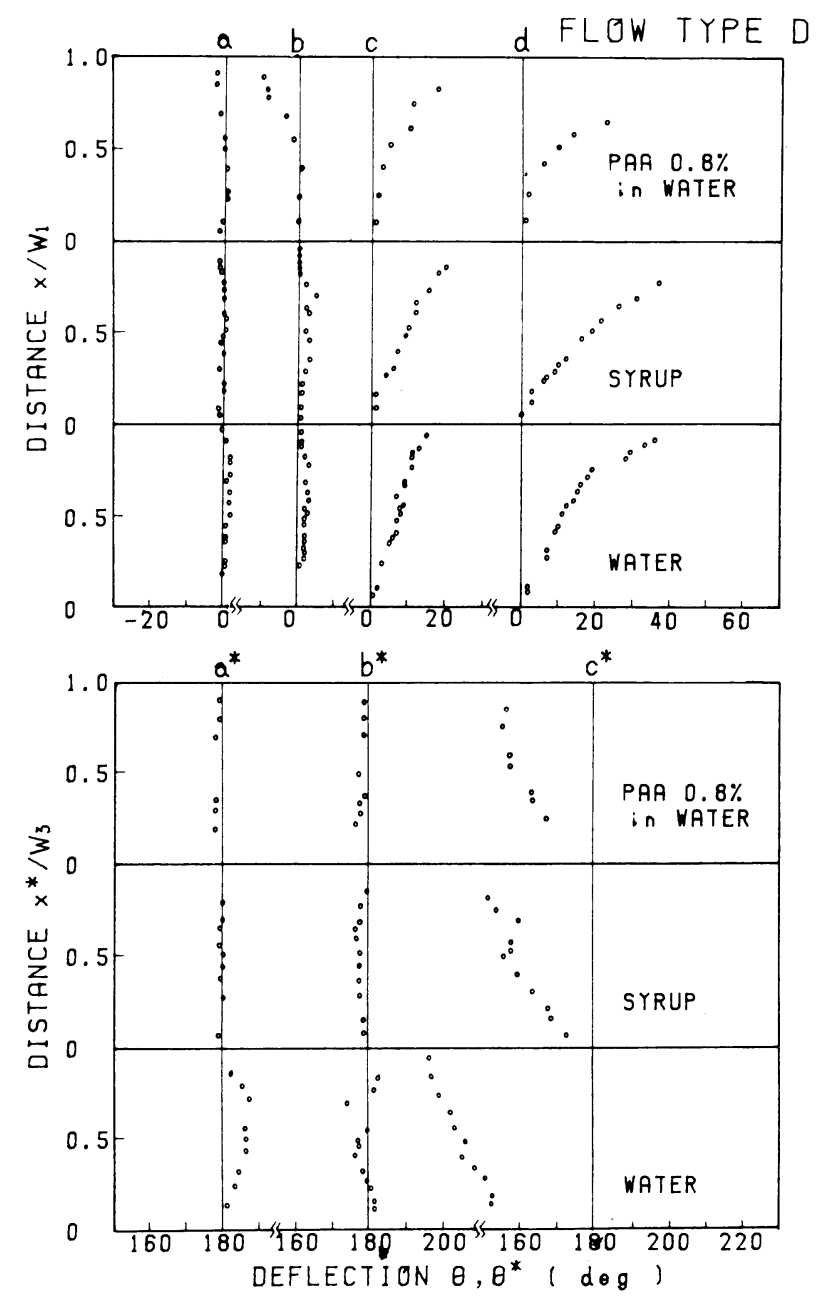

Fig. 6 Experimental flow direction ditributions for asymmetric branch flows. 
by the authors. ${ }^{[1]}$

Photographs [3] and [4] in Fig. 5 show flows of Newtonian dextrose syrup and water. A secondary flow due to inertial effect was observed on the downstream corner in the water flow at a high Reynolds number shown in Fig. 5 [4].

When flow rate is low and Reynolds number small a secondary flow was not observed with both Newtonian and viscoelastic fluids as photographs [2] and [3] show.

D. Flow directions in asymmetric branch flows

Flow directions read off photographs [1], [3] and [4] in Fig. 5 are shown graphically in Fig. 6. The flow of PAA solution in photo [1] and that of water in photo [4] were large in Reynolds number while the flow [3] of dextrose syrup had small Reynolds number.

Before branching the three flows [1], [3] and [4] are similar in flow direction distribution curves. After branching, however, the flow of viscoelastic PAA solution is different from the other two flows in that the flow direction angle has negative values on line $b$ near the corner. On line $c$, the flow detection curve for the viscoelastic fluid is similar to those of two Newtonian fluids. On line d near channel 3, the viscoelastic flow [1] turns to the exit more abruptly than the Newtonian fluids.

After branching the flow direction curves for PAA solution in channel 3 are similar to the small Reynolds Newtonian flow [3] with angle $\theta^{*}$ values on line $\mathrm{a}^{*}$ and $\mathrm{b}^{*}$ being approximately $180^{\circ}$. On line $c^{*}$, however, the $\theta^{*}$ values for PAA solution are closer to $180^{\circ}$ than with the syrup. Those results suggest that the viscoelastic flow turns more abruptly in flowing from channel 1 into 3 than in Newtonian fluids as was the case in branching flows. The water flow [4] is large in Reynolds number, angle $\theta^{*}$ on line $a^{*}$
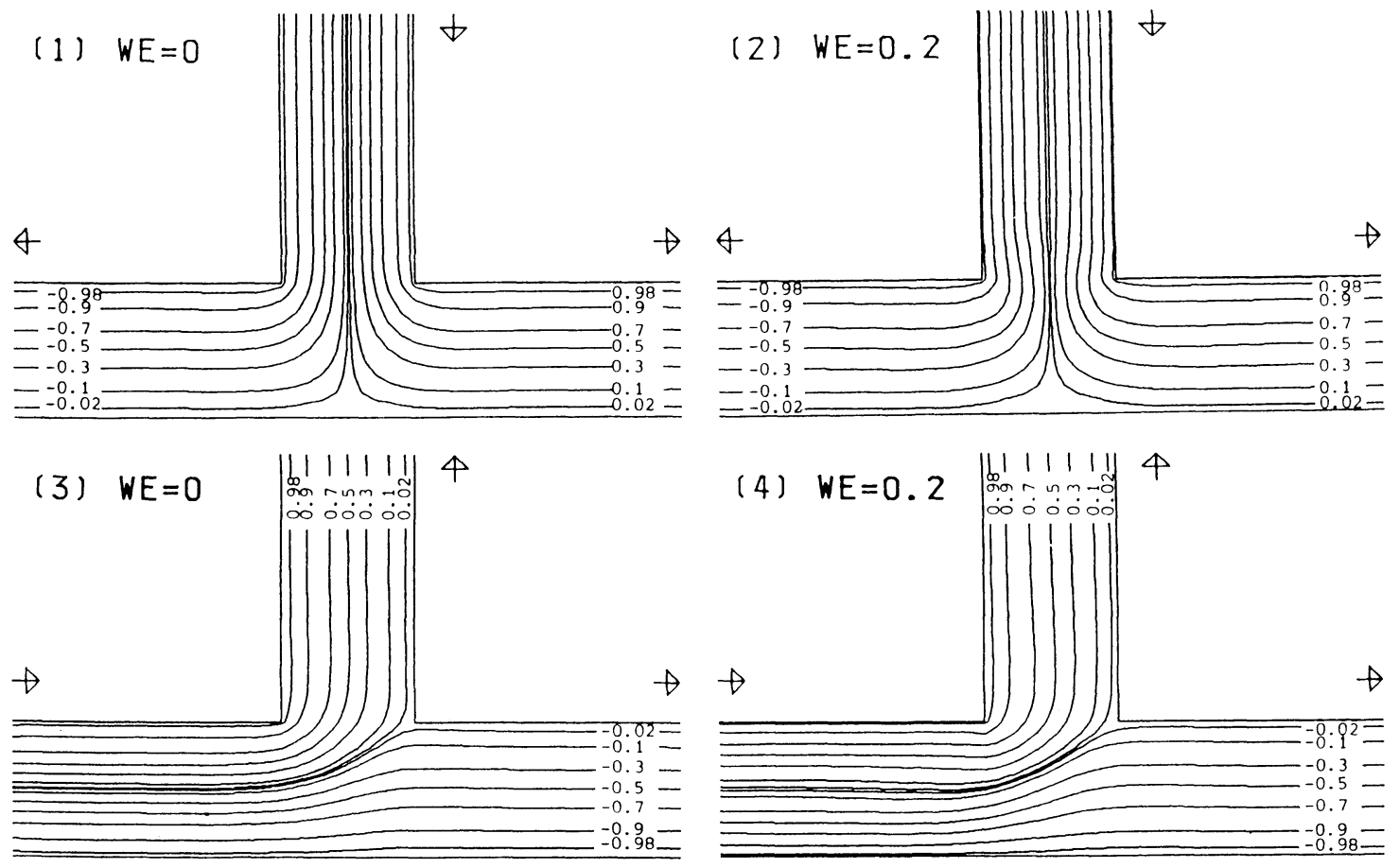

Fig. 7 Streamlines predicted numerically: symmetric branch flow [1] at Weissenberg number $=0$ and [2] at Weissenberg number $=0.2$, and asymmetric branch flow [3] at Weissenberg number $=0$ and [4] at Weissenberg number $=0.2$.

are larger than $180^{\circ}$ and on line $c^{*}$ angle $\theta^{*}$ is smaller than with two other fluids because of the presence of the secondary flow due to inertia at the downstream corner.

\subsection{Numerical simulation}

Streamlines for the symmetrical and asymmetrical branch flows were numerically simulated using the Maxwell constitutive model in perturbation method. ${ }^{[2]}$ Inertial terms in the governing equations were ignored because of the lower flow rate and high viscosity. Details of the analysis were described previously. ${ }^{[1]}$

\section{A. Streamlines}

Show in Fig. 7[1] and [2] are contours of constant stream function values computed for symmetric branch flow and those in Fig. 7[3] and [4] are for asymmetric branch flows. Comparison of flow lines in Fig. 7[2] and [4] with those in Figs. 7[1] and [3] reveals that viscoelasticity tends to make the flow line just upstream of the corner slightly bulged. This tendency agrees with experimental results.

Flow direction distributions thus numerically predicted are shown graphically in Figs. 8 and 9. Lines a, b, c, etc., distances $x, x^{*}$ and angles $\boldsymbol{\theta}, \boldsymbol{\theta}^{*}$ are as indicated in Fig. 3 . Shown in Fig. 8 are numerically predicted flow direction distributions for symmetric branching flows. In the viscoelastic flow [2] of $W_{e}=0.2$ flow lines at $\mathrm{a}^{*}, \mathrm{~b}^{*}$ and $\mathrm{c}^{*}$ bend toward the center (note that the $\boldsymbol{\theta}^{*}$ scale on line $\mathrm{a}^{*}$ is magnified by ten times). After branching the viscoelastic flow [2] is similar to the Newtonian flow [1] excepe that the $\theta$ values on line $\mathrm{c}$ are closer to $180^{\circ}$ than with Newtonian fluids. These numerical results are in agreement with the experimental observations that viscoelastic flows turn more abruptly at the channel corner than Newtonian flows.

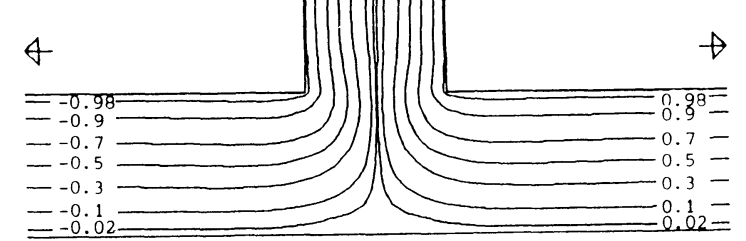

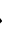

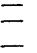

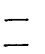
(1) 


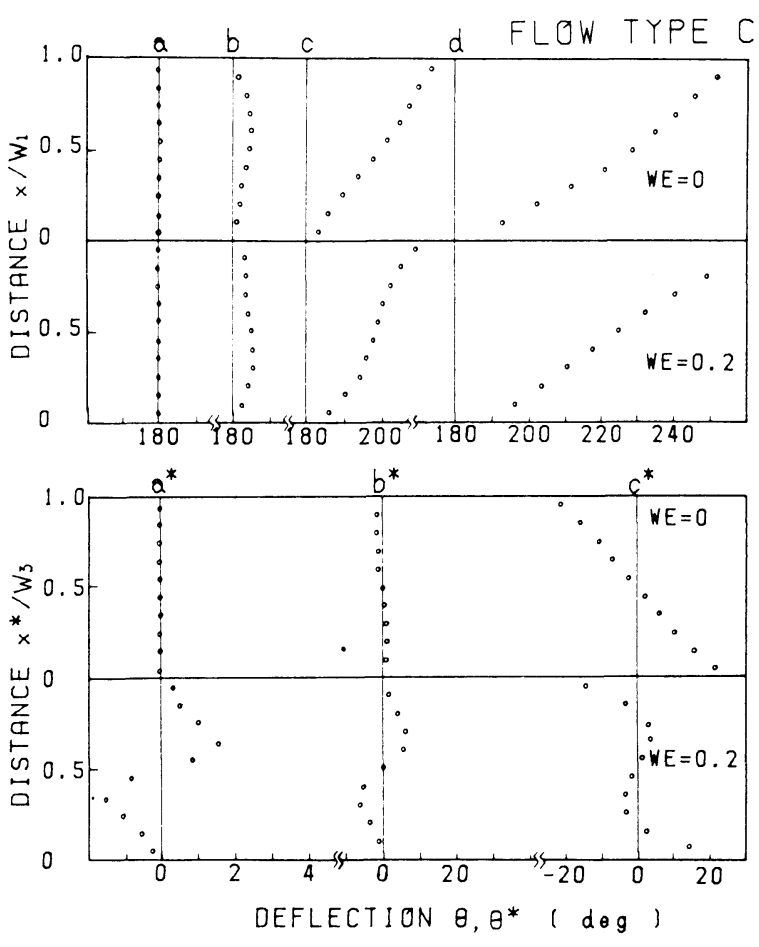

Fig. 8 Flow direction distributions predicted numerically for symmetric branch flow.

Fig. 9 shows flow direction distribution for the two asymmetric flows. On lines a, b, $\mathrm{c}$ and $\mathrm{d}$ before branching, angle $\theta$ at $x / W_{1}=0.6-0.7$ are smaller for the viscoelastic flow [4] than for the Newtonian flow [3], probably due to the existence of a circulating secondary flow at the upstream corner in the viscoelastic flow [4]. Newtonian and viscoelastic flows differ little in the flow direction distribution
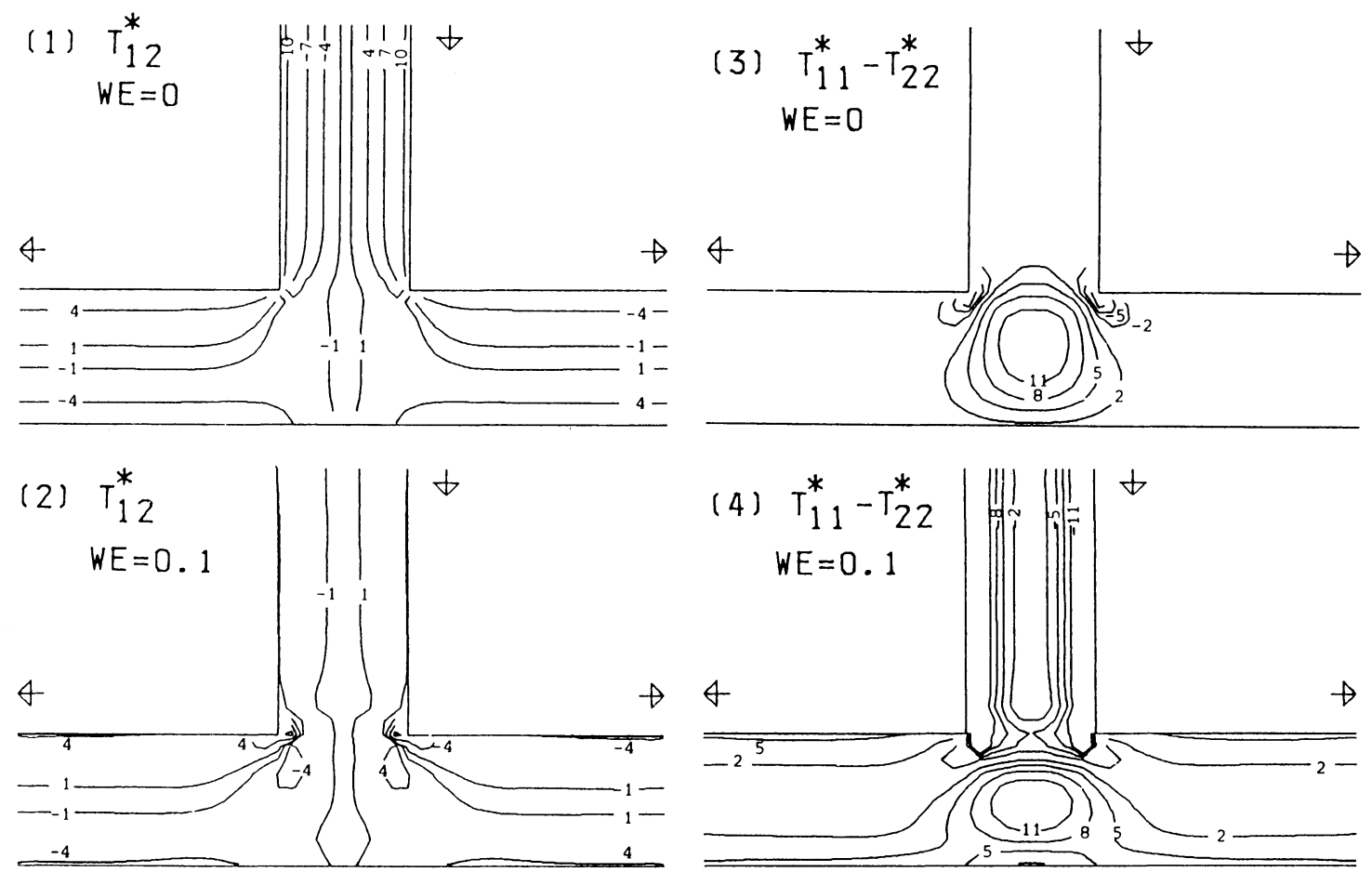

after branching except that on line $\mathrm{b}$ angle $\theta$ is closer to $180^{\circ}$ in the viscoelastic flow than in the Newtonian flow. Numerical analysis thus predicts that viscoelastic flow changes direction more abruptly at the corner than the Newtonian flow like in symmetric branch flows and this prediction agrees with experimental results shown in Fig. 6.

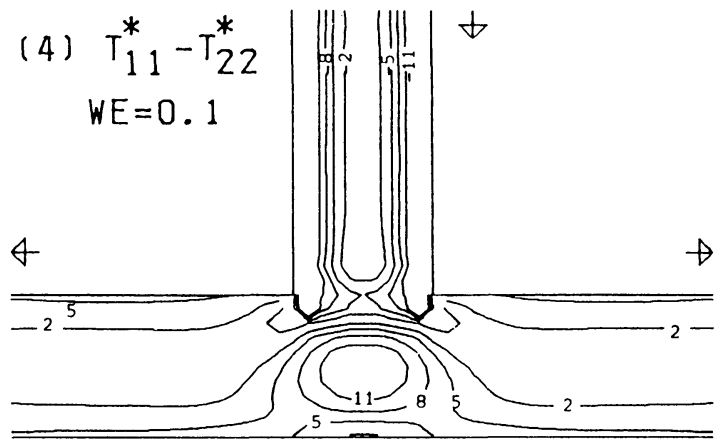

Fig. 10 Numerically predicted stress distributions for symmetric branch flows: non-dimensional shear stress distributions for flow [1] at Weissenberg number $=0$ and for flow [2] at Weissenberg number $=0.1$, and nondimensional normal stress distributions for flow [3] at Weissenberg number $=0$ and for flow [4] at Weissenberg number $=0.1$. 


\section{B. Stress distribution}

Numerically simulated stress distribution for symmetric branch flow are shown in Fig. 10. Figures 10[1] and [2] respectively show simulated distribution of non-dimensional shear stress $T *_{12}$ at the Weissenberg number values of $W_{e}=0$ and 0.1 , while [3] and [4] represent non-dimensional normal stress differences $T{ }^{*}{ }_{11}-T *_{22}$ at $W_{e}=0$ and 0.1 . Generally the shear stress decreases with increasing Weissenberg number except at the channel corner where the shear stress increases with increasing Weissenberg number. Contours of constant normal stress difference become more closely spaced and the center peak near the branch becomes deformed as the Weissenberg number increases.

Shown in Fig. 11 are numerically simulated stress distributions for the asymmetric branching flows. Figure 11 [1] and [2] show non-dimensional shear stress, and [3] and [4] show non-dimensional normal stress differences. While symmetry is lost in contour patterns, qualitative effects of elasticity are similar to that in symmetric branch flows.

\section{Pocker Flow}

\subsection{Experimental results}

\section{A. Streamlines of L flows}

Experimental observations of $\mathrm{L}$ flows are shown in Figs. 12. In $\mathrm{L}$ flow the fluid enters from channel 3 and exits to channel 2 while the far end of channel 1 is closes. Figures 12[1], [2] and [3] show flows of PAA $0.8 \%$ solution with the velocity increasing in that order. Difference between the viscoelastic flows [1]-[3] and the Newtonian flow [4] with dextrose syrup become pronounced with increasing flow rate.
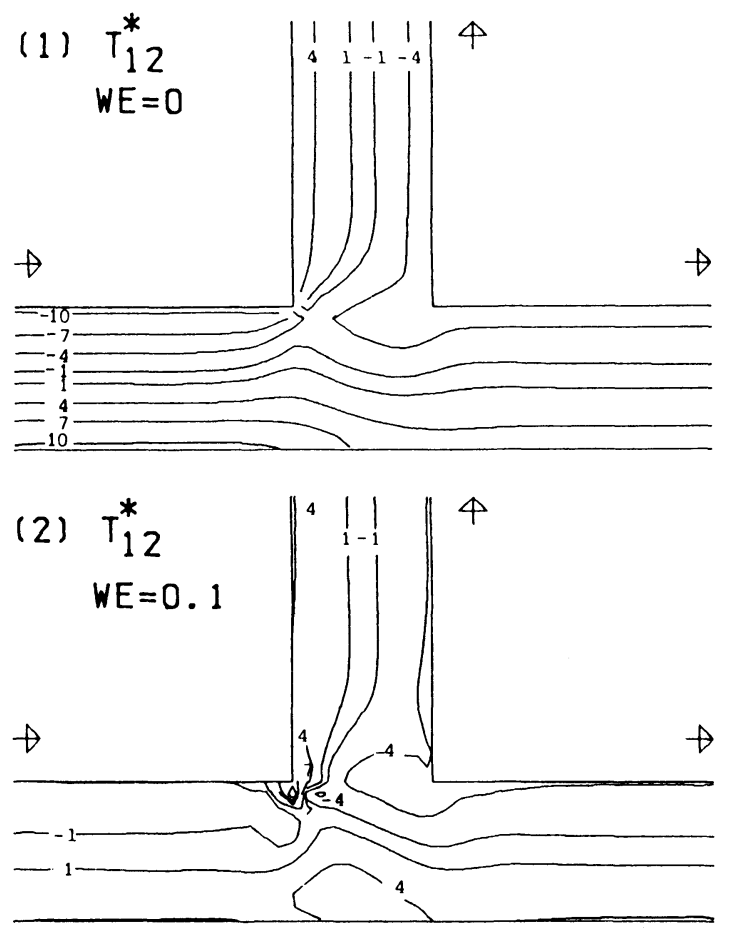
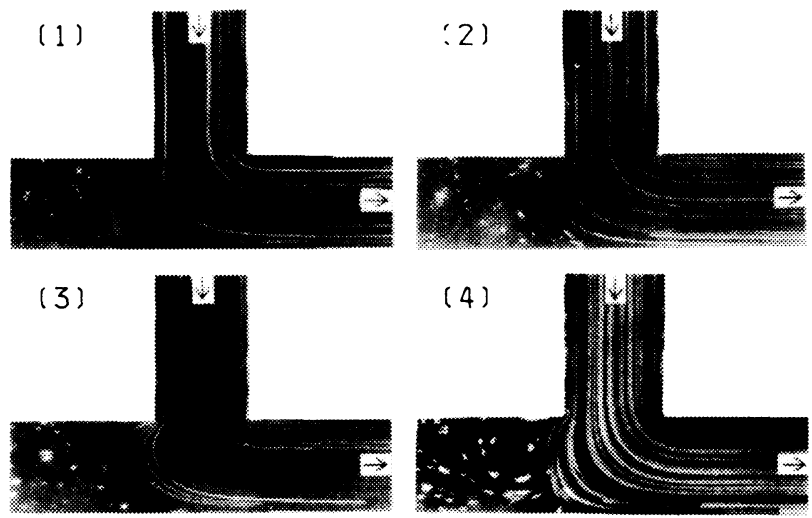

Fig. 12 Experimentally observed flow patterns for L flows: [1] $\mathrm{PAA}, V=10 \mathrm{~cm} / \mathrm{min}, R e=6.9 \times 10^{-3}$, [2] PAA, $V=50 \mathrm{~cm} /$ $\min , R e=9.7 \times 10^{-2}$, [3] PAA, $V=500 \mathrm{~cm} / \mathrm{min}, R e=5.2$, [4] syrup, $V=50 \mathrm{~cm} / \mathrm{min}, R e=8.7 \times 10^{-2}$.
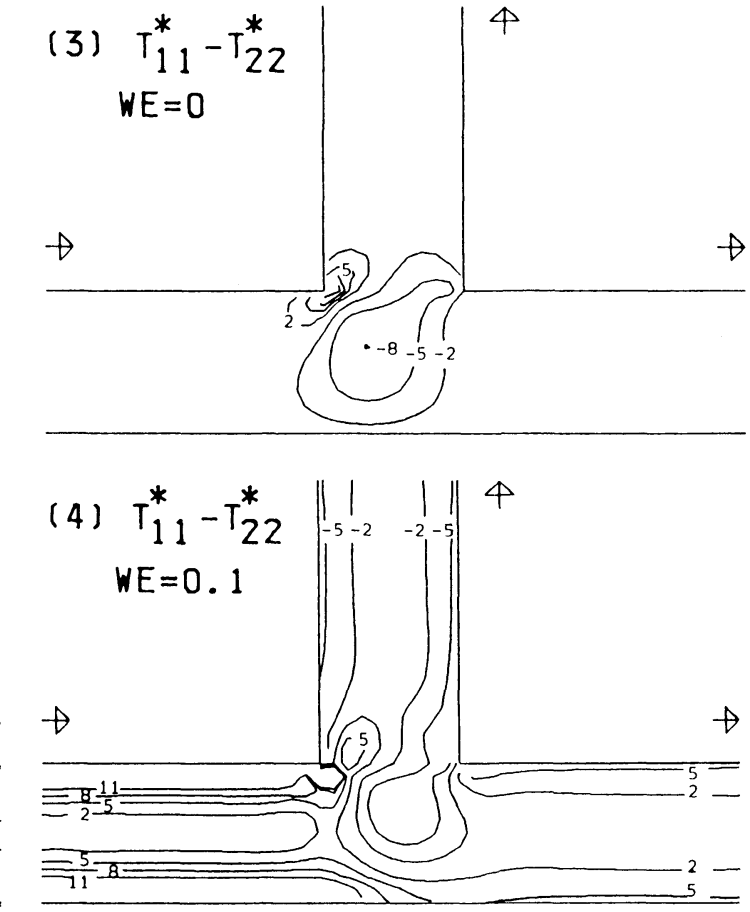

Fig. 11 Numerically predicted stress distributions for asymmetric branch flows: non-dimensional shear stress distributions for flow [1] at Weissenberg number $=0$ and for flow [2] at Weissenberg number $=0.1$, and nondimensional normal stress distributions for flow [3] at Weissenberg number $=0$ and for flow [4] at Weissenberg number $=0.1$. 
other flows to obtain detailed information on viscoelastic effects. Measurements were taken at the broken lines specified in Fig. 3.

The measured flow direction distribution are shown in Fig. 13. Figure 13[4] is flow direction distribution for the Newtonian flow, and [1] is that of viscoelastic flow at a low flow rate, and [3] is the same at a high flow rate. The distributions were read off the photographs in Fig. 12[4], [1] and [3] respectively.

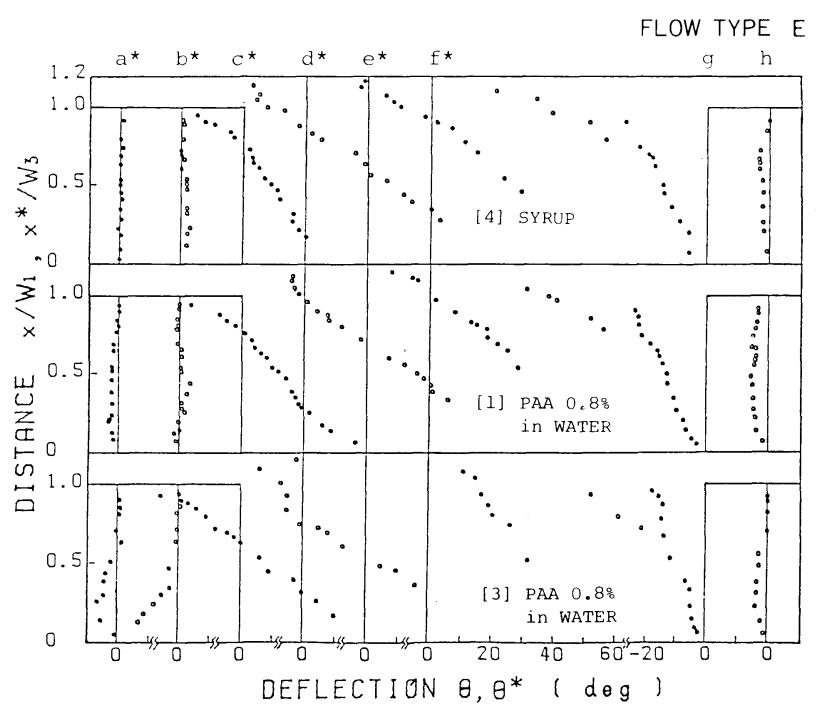

Fig. 13 Experimental flow direction distribution for L flows.

In flow [3] angle $\boldsymbol{\theta}^{*}$ is negative on lines $\mathrm{a}^{*}$ and $\mathrm{b}^{*}$ near the wall at $x^{*} / W_{3}=0$ reflecting the swell of the circulating secondary flow at the upstream corner. The $\theta^{*}$ values on line $c^{*}$ near the wall $x / W_{3}=1$ is smaller in viscoelastic flow [3] than in Newtonian flow [4]. Moreover, the $\boldsymbol{\theta}^{*}$ value for viscoelastic flows decreases with increasing flow rate. This means that the outermost streamline bends more abruptly toward channel 1 with increasing elastic effect of the flow. Values of $\boldsymbol{\theta}^{*}$ on line $\mathrm{e}^{*}$ increase in the order of Newtonian flow [4], slow viscoelastic flow [1] and fast viscoelastic flow [3]. This is to mean that the viscoelastic flows bulge into the dead pocket more extensively than Newtonian flow but recover form the bulge more quickly.

On line $g$ viscoelastic flows are aligned closer to the exit direction since $\theta$ is closer to zero than with Newtonian fluid. On line h values of angle $\theta$ are negative in Newtonian flow at large $x / W_{1}$ values while with viscoelastic fluids $\theta$ is negative when $x / W_{1}$ is small. The mean value of $\boldsymbol{\theta}$ on line $\mathrm{h}$, however, does not differ greatly by the two fluids.

C. Streamlines of reverse $L$ flows

Reverse L flows shown in Figs. 14 flow from channel 1 to channel 3 while the far end of channel 2 is closed to form a pocket. Figures 14[1], [2] and [3] are the streamlines for the viscoelastic PAA solution with the flow rate increasing in that order. Flow [4] is for the Newtonian dextrose syrup.

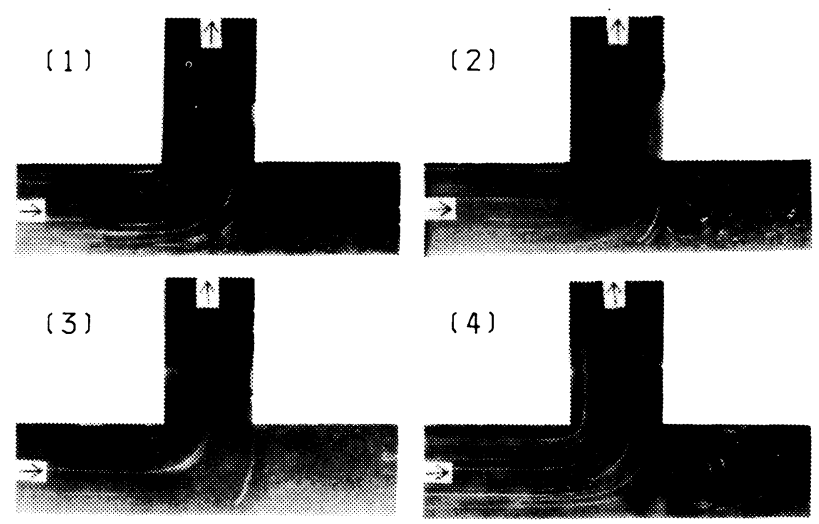

Fig. 14 Experimentally observed flow patterns for reverse $\mathrm{L}$ flows: [1] PAA, $V=1.0 \mathrm{~cm} / \mathrm{min}, R e=2.3 \times 10^{-4}$, [2] PAA, $V=22 \mathrm{~cm} / \mathrm{min}, R e=2.8 \times 10^{-2}$, [3] PAA, $V=150 \mathrm{~cm} / \mathrm{min}$, $R e=6.0 \times 10^{-1}$, [4] syrup, $V=10 \mathrm{~cm} / \mathrm{min}, R e=1.7 \times 10^{-2}$.

Viscoelastic flows are different from Newtonian flows in two respects. One is the formation of a circulating secondary flow on upstream corner as shown in Fig. 14[3]. The circulating flow grows with increasing flow rate as was the case in merging and branching flows. Second is the bulging of outer stream lines into the blocked channel 2. The bulge is present both in Newtonian flow [4] and low speed viscoelastic flow [1] but in the fast viscoelastic flow [3] the bulge is absent and the bending of flowlines is more abrupt than in Newtonian flows.

\section{Flow direction of reverse $\mathrm{L}$ flows}

Shown in Fig. 15 are flow direction distributions in reverse $L$ flows. Figure 15[4], [1] and [3] were obtained by reading $\theta$ and $\theta^{*}$ off the photographs in Fig. 14[4], [1] and [3] showing respectively Newtonian, slow viscoelastic and fast viscoelastic flows.

The Newtonian flow [4] starts to bend toward the exit well upstream of the bend. This is indicated by the positive $\theta$ values on line b. Whereas the slow viscoelastic flow [1] exhibits no such early bending. The fast viscoelastic flow [3] on the other hand exhibits negative $\theta$ values on line b

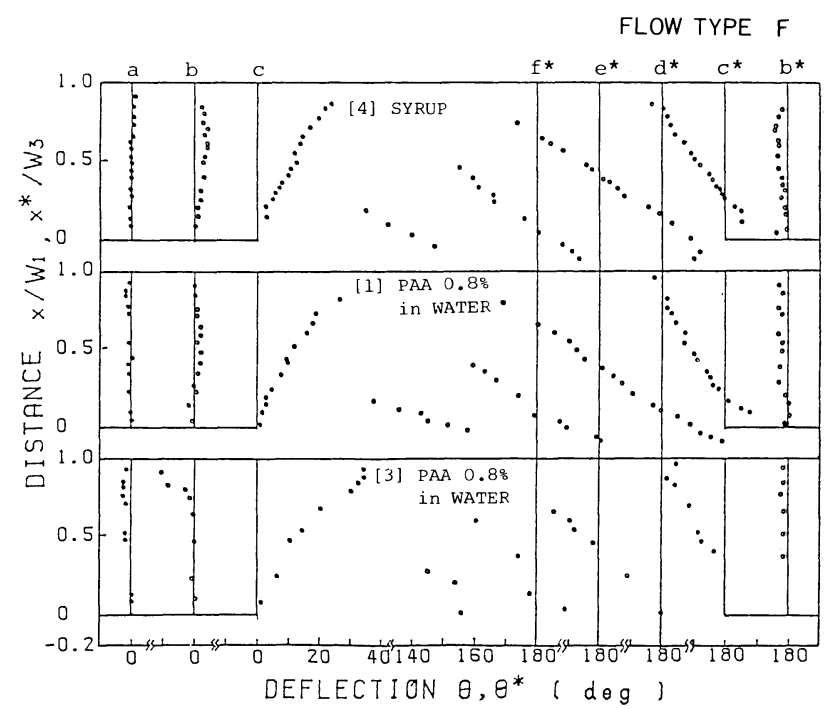

Fig. 15 Experimental flow direction distributions for reverse $\mathrm{L}$ flows. 
near the wall at $x / W_{1}=1$ indicating that the flow turns to opposite direction before making the right angle turn. This is due to the circulating secondary flow.

Viscoelastic flows [1] and [3] are larger in $\theta$ values on line $\mathrm{c}$ and closer to $180^{\circ}$ on line $\mathrm{f}^{*}$ than the Newtonian flow [4]. This is an indication that viscoelastic flows make more abrupt turns at the corner than Newtonian flows.

On line $\mathrm{e}^{*}$ value of $\boldsymbol{\theta}^{*}$ for viscoelastic flows are closer to $180^{\circ}$ than that of Newtonian flow. The $\theta^{*}$ on line $\mathrm{e}^{*}$ tends to zero at $x * / W_{3}=0$. Evidently viscoelastic flows tend not to bulge into the blocked channel 2 . On line $\mathrm{d}^{*}$ angles $\boldsymbol{\theta}^{*}$ at $x^{*} / W_{3}=0$ become smaller in the order Newtonian flow [4], slow viscoelastic flow [1], and fast viscoelastic flow [3] being about $180^{\circ}$ on flow [3]. The $\boldsymbol{\theta}^{*}$ values larger than $180^{\circ}$ for [4] and [1] reflect the swell of flowlines into channel 2. Flow [3] does not exhibit such swell. These results are consistent with the findings in the above $4.1 \mathrm{C}$.

\section{E. Streamlines on "Straight" flows}

Show in Fig. 16 are photographs of "straight" flows flowing from channel 1 to channel 2 past the blocked channel 3. Figure 16[1], [2] and [3] are viscoelastic flows with velocities increasing in that order and [4] is Newtonian flow. Both Newtonian and viscoelastic flow swell into the blocked channel 3. While the swelling is symmetric in Newtonian flows it is asymmetric in viscoelastic flows with the streamlines quickly expanding into channel 3 but contracting slowly. The swell of viscoelastic flows diminish with increasing flow rate but the asymmetry remains.
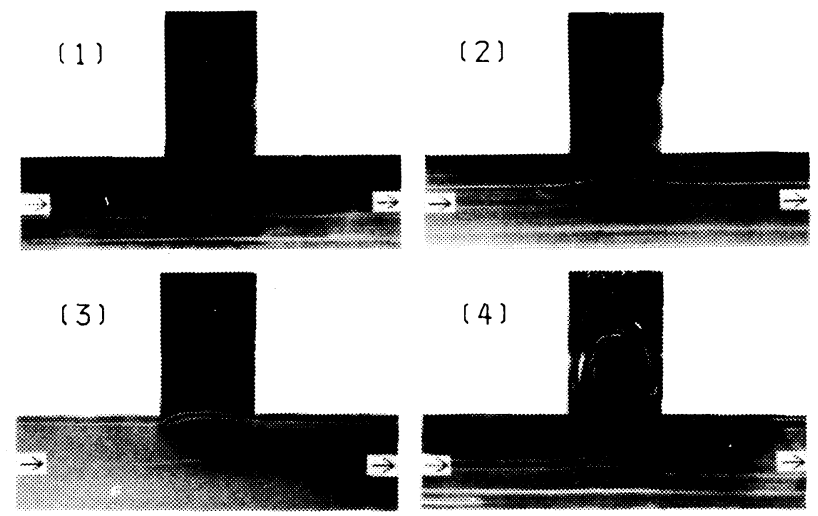

Fig. 16 Experimentally observed flow patterns for straight flows: [1] PAA, $V=1.5 \mathrm{~cm} / \mathrm{min}, R e=3.8 \times 10^{-4}$, [2] PAA, $V=26 \mathrm{~cm} / \mathrm{min}, R e=3.3 \times 10^{-2}$, [3] PAA, $V=430 \mathrm{~cm} / \mathrm{min}$, $R e=3.8 \times 10^{-1},[4]$ syrup, $V=50 \mathrm{~cm} / \mathrm{min}, R e=8.7 \times 10^{-2}$.

\section{F. Flow direction of "Straight" flows}

Flow direction distributions for "straight" flows are shown in Fig. 17. Figure 17[4] is flow direction distributions for the Newtonian flow, and [1], [3] are for viscoelastic fast flow and slow flow read off the photographs in Fig. 16[4], [1] and [3] respectively.

In the Newtonian flow [4] the angle $\theta$ versus distance $x / W_{1}$ plots shown in Fig. 17[4] are symmetrical with respect to line e on which $\theta$ values are approximately equal to zero. Whereas in viscoelastic flows [1] and [3] negative $\boldsymbol{\theta}$ values are observed on the center line e and $\boldsymbol{\theta}$ is larger in lines $c$ and $d$ than on $g$ and $f$ to reflect the skewed

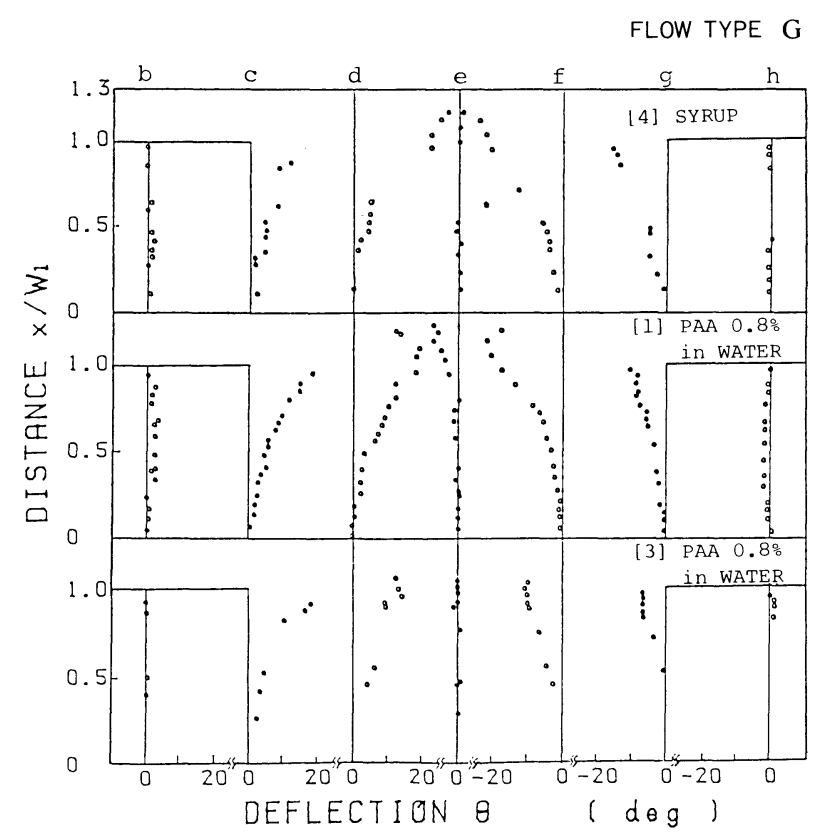

Fig. 17 Experimental flow direction distributions for straight flows.

flowline bulge in the pocket channel 3 .

The bulge of viscoelastic flow diminishes and the asymmetry of the bulge becomes insignificant with increasing flow rate. As a result $\theta$ values on lines $d$ and $\mathrm{f}$ in Fig. 17[3] near the $x / W_{1}=1$ line are smaller than in Fig. $17[1]$.

\subsection{Numerical simulation}

\section{A. Predicted streamlines}

Streamlines for pocket flows predicted numerically agreed qualitatively with experimental results. For L flows, swelling of streamlines at the upstream corner was predicted by the viscoelastic fluid model although not to the extent of the experimental observations. As in experiments viscoelastic fluids aligned toward the exit in L flows more quickly than Newtonian fluids. Numerical simulation, however, did not predict the bulging of fast viscoelastic flow into the blocked channel.

For reverse $\mathrm{L}$ viscoelastic flows swelling of streamlines at the upstream corner was simulated as in other viscoelastic flows. As in experiments reverse $\mathrm{L}$ viscoelastic flows bulged into the blocked channel 2 to a less extent than Newtonian flows.

In "straight" flows magnitude of the swelling of streamlines into the blocked channel decreased with increasing Weissenberg number to agree with the experiments shown in Fig. 16, but the asymmetry of the swell was not simulated numerically.

\section{B. Stress distribution}

Contours of constant non-dimensional shearing stress for pocket flows L, reverse L and "straight" all become more sparsely spaced with increasing Weissenberg number but in $\mathrm{L}$ and reverse $\mathrm{L}$ flows the contour spacing became denser at the inside corner.

The values of non-dimensional normal stress difference decreased at stream center with increasing Weissenberg number. While at other areas of the channel the normal 
stress difference increased and its contours became more close together. This was particularly the case at inside corner in $\mathrm{L}$ and reverse $\mathrm{L}$ flows and at both channel corners in reverse $\mathrm{L}$ and "straight" flows.

\section{Conclusions}

Two kinds of branch flows and three kinds of pocket flows in T-shaped channels were observed experimentally to compare vicoelastic flows with Newtonian flows.

A circulating secondary flow was observed at the upstream channel corner with viscoelastic polymer solutions. The secondary flow appeared at slower flow speed in merging flows than in $\mathrm{L}$ and reverse bend flows while highest flow speed was required to form secondary flows in branching flows.

When the three channels were different in width in $\mathrm{T}$ shaped symmetric branching flows the narrower the exit channel the lower was the entrance flow speed at which the secondary flow appeared.

In L and "straight" flows both Newtonian and viscoelastic fluids exhibited swell of streamlines into the blocked pocket channel. With viscoelastic fluids, however, the expansion side of the swell was steeper than the contraction to make the swell asymmetric while Newtonian fluids exhibited symmetric swell.

In reverse L and "straight" flows the swell of flowlines into the blocked channel diminished with increasing elasticity (or Weissenberg number).

Photographed streamlines and the plot of flow direction distribution read off the photographs revealed that streamlines of viscoelastic fluids turned at the channel corner more abruptly than with Newtonian fluids.

Above flows were numerically simulated by expanding the Maxwell constitutive equation into a power series of Weissenberg number. The circulating secondary flow was not simulated, but a swelling of streamlines suggesting the existence of secondary flow appeared at the upstream channel corner where the secondary flow was observed experimentally.

In both reverse L and "straight" flow the swell of simulated streamlines into the blocked channel diminished with increasing elastic effect to agree with experimental results. However, the abrupt expansion of flowlines into the stagnant channel in L flow and the asymmetric swell in "straight" flow observed on viscoelastic fluids were not predicted numerically.

\section{References}

[1] T. Nishimura, K. Nakamura and A. Horikawa; Sen-i Kikai Gakkaishi, 36, T66 (1983).

[2] K. Nakamura, T. Azuma and A. Horikawa; Sen-i Kikai Gakkaishi, 30, T167 (1977). 